\title{
ROCK DEN BALKAN! \\ Die mUSikalische ReKonstruktion des BALKanS \\ ALS EMOTIONALES TERRITORIUM
}

\author{
Alenka Barber-Kersovan
}

\section{»Reichtum der Unterschiede«}

Seit dem Anfang des neuen Jahrtausends verzeichnet man ein zunehmendes Interesse am Balkan. Davon zeugen sowohl politische Gesprächsrunden und wissenschaftliche Tagungen als auch zahlreiche Kunstereignisse wie etwa die Ausstellungen Schluchten des Balkan (Kassel, 2003), In Search of Balkania (Graz, 2002) oder Blut \& Honig. Zukunft ist am Balkan (Klosterneuburg bei Wien, 2003). Auch im popmusikalischen Bereich häufen sich Tonträger mit Titeln wie Balkan, Balkan Brass Fest, Trans Balkan Express, Balkan Messengers oder Sketches of Balkan. Dieses musikalische Balkan-Fieber, das besonders stark Österreich und die Bundesrepublik Deutschland erfasste, grassiert unter mehreren Bezeichnungen, unter denen »Balkan-Rock«, »Balkan-Beat « und - etwas seltener - »Balkan-Jazz« die häufigsten sind.

Der Begriff Balkan-Rock wurde zunächst auf den einst florierenden Yugo-Rock angewandt. Anders als in den anderen Balkanländern (Bulgarien, Rumänien, Albanien) existierte nämlich in Ex-Jugoslawien eine äußerst lebendige Rockszene (vgl. Ramet 1994; Janjatovic 1998), die vom sozialistischen Regime nicht nur toleriert, sondern sogar ausdrücklich gefördert wurde - nicht zuletzt auch deshalb, weil man durch die Anlehnung an die westliche Popkultur erhoffte, sich einerseits merklich vom Ostblock abzugrenzen und andererseits die bestehenden Differenzen zwischen den einzelnen ethnischen Gruppierungen einzuebnen. Ganz überdecken ließen sich diese damals als Reichtum bewerteten (kulturellen) Unterschiede nicht und dementsprechend waren auch die Szenen in den einzelnen Republiken unterschiedlich gelagert. In Mazedonien und in Montenegro wurde die Rockmusik nur sporadisch gepflegt. Kroatien hingegen besaß eine hoch entwickelte Musikindustrie und aus Zagreb stammten die professionellsten 
Erzeugnisse der jugoslawischen Popmusik. Aushängeschilder der vorwiegend rockorientierten Szene von Beograd waren die Bands Disciplina kicme (Die Disziplin des Rückgrates) und Ekaterina velika (Katharina die Große). Die Alternativszene von Ljubljana wiederum wurde vor allem durch den politisch gefärbten Punk und die Skandalgruppe Laibach bekannt.

Es gab aber auch verbindende Elemente. Die kommerziell orientierte, obwohl im Gegensatz zu den anderen sozialistischen Ländern weitgehend dezentralisierte Musikindustrie ging vom gemeinsamen Binnenmarkt aus: Bekannte Rockgruppen tourten in ganz Jugoslawien; Sänger wie Miroslav Ilic ${ }^{1}$, Ljubka Dimitrova oder Zdravko Colic wurden in allen Landesteilen als Stars gefeiert; an den zahlreichen Musikfestivals und Wettbewerben nahmen Musiker aus dem ganzen Staatsgebiet teil; die als national betrachteten Fernsehsender übertrugen auch populäre Musiksendungen aus den anderen Republiken etc. Und schließlich gab es den Yugo-Rock, einen Hybrid zwischen der internationalen Popmusik und den heimischen Musiktraditionen, der die Fans über alle Grenzen hinweg begeisterte.

\section{Bijelo dugme und der Yugo-Rock}

Die Wiege des Yugo-Rock war Sarajevo. Vor dem Bürgerkrieg 1992-1995 wurde Sarajevo aufgrund seiner multikulturellen sozialen Struktur als Jugoslawien im Kleinen, wenn nicht sogar als eine holographische Abbildung des Balkans insgesamt betrachtet. Die Stadt war ein lebendiges, tolerantes und inspirierendes urbanes Zentrum, in dem serbische, kroatische und muslimische Traditionen aufeinander trafen und sich mit diversen Strömungen der internationalen Popmusik zu einem eigenständigen Musikidiom verbanden (vgl. Anon. 1991/92).

Als Begründer des Yugo-Rock gilt die bosnische Gruppe Bijelo dugme (Der weiße Knopf). Ihnen gelang als Ersten auf überzeugende Art und Weise, den »Geist des Rock'n'Roll« mit der »bosnischen Mentalität« zu fusionieren. Dabei besaß nicht nur der Hard-Rock-Sound dieser an Deep Purple anknüpfenden Band ein unverkennbar lokales Kolorit. Auch ihre Texte, getragen von geläufigen Sinnbildern und Vergleichen, spiegelten die unmittelbare Volksnähe wider, so dass man mit Gregor Tomc (1998: 41) ihre Musik als »balkanischen Country Rock« bezeichnen kann.

Ein weiterer bedeutender Vertreter dieser Stilrichtung in den 1980er Jahren war die Gruppe Plavi orkestar (Das Blaue Orchester), die ebenfalls aus Sarajevo stammte. Ihre Musik wurde aufgrund zahlreicher Referenzen

1 Auf diakritische Zeichen wird aus technischen Gründen durchgehend verzichtet. 
an den Balkanmelos auch unter den Begriffen »balkanischer Folklorismus«, »neuer Primitivismus« bzw. »neukomponierter Poprock« bekannt (Gracanin 1985: 47). »Wir sind volkstümliche Musiker«, erklärte der Sänger in einem Interview:

»Hier gibt es keinen ironischen Abstand zum Volk. Wir verherrlichen das Volk und die Volkskultur. Wir zahlen unsere Steuer an die Tradition. Alle originellen Ideen sind heimischer Herkunft. Bei uns handelt es sich um die vollkommene Annahme der revolutionären Vergangenheit, der kulturellen und vor allem der nationalen Vergangenheit, des Volksschatzes« (Sasa Losic, zit. n. Gracanin 1987: 44; übers. aus dem Slowenischen v. d. Verf.).

Ein weiteres Merkmal war ihr »eher emotionales Verhältnis« zur Musik, das »lebensbejahend bzw. optimistisch war und viel mit Humor operierte « (Barbabegovic 1984: 38/40; übers. aus dem Slowenischen v. d. Verf.). Ähnlich wie Produktionen von Bijelo dugme waren deshalb auch Songs dieser als »die balkanischen Beatles« gepriesenen Gruppe nicht nur in urbanen, sondern auch in ruralen Gegenden äußerst beliebt (vgl. Virant 1998: 25).

\section{Desintegration der Musikszene und die integrative Kraft des Yugo-Rock}

Mit den aufkommenden nationalen Spannungen begann sich - ähnlich allen anderen Lebensbereichen - ab Mitte der 1980er Jahre auch die populäre Musik in den jeweiligen republikanischen bzw. ethnischen Grenzen abzukapseln. Multinationale Gruppen zerfielen, gesamtjugoslawische Großevents verloren ihre Bedeutung (vgl. Janjatovic 1998), die Medien sendeten vorwiegend angloamerikanische Popmusik und Titel lokaler bzw. regionaler Gruppen, und Musiker, die einst im ganzen Land präsent waren, mussten ihre Tätigkeit auf das eigene kulturelle Umfeld beschränken. Die einst multikulturelle Musiklandschaft zerfiel in eine Reihe von ethnisch bestimmten musikalischen Monokulturen mit stärkerer oder schwächerer Anlehnung an internationale Trends.

Musiker reagierten auf diese Situation durchaus unterschiedlich. Viele gingen, angewidert von den politischen Ereignissen, ins Ausland, vor allem nach London, Amsterdam und Berlin, wo es starke Communities musikalischer Emigranten aus allen Teilen des ehemaligen Jugoslawien gibt. Andere blieben und ließen sich - insbesondere in Serbien und Kroatien - für politische Zwecke instrumentalisieren. Es gab aber auch einige, die aus Protest ihren Beruf änderten oder sich an Anti-Kriegs-Demonstrationen beteiligten. 
$\mathrm{Zu}$ jenen, mit denen die nationalistischen Machthaber »nicht rechnen konnten ${ }^{2}$ bzw. die bereits im Vorfeld der Kriegshandlungen vor einer Eskalation ethnischer Konflikte warnten, gehörten auch Bijelo dugme. Die Gruppe verschrieb sich einer Art jugoslawischem Nationalismus und nahm unter anderem eine Coverversion der einstigen jugoslawischen Staatshymne auf. Sie glaubte an die integrative Kraft des Yugo-Rock und versuchte - wie etwa im Song »Pljuni i zapjevaj, moja Jugoslavijo« (Spuck und sing, mein Jugoslawien) aus der 1986 veröffentlichten gleichnamigen LP - mit ihrer Musik den Bürgerkrieg zu verhindern (vgl. Tomc 1998: 41). In diesem Lied betete die Gruppe das »Mädchen Jugoslawien« an und appellierte an ihre sich damals bereits in einer schweren Agonie befindende Geliebte: "Steh auf, mein geliebtes Jugoslawien, und sing. Wer dem Gesang nicht lauscht, wird den Donner hören« (übers. aus dem Serbokroatischen v. d. Verf.).

Auch Plavi orkestar gab 1986 eine ähnlich konzipierte LP heraus. Ihr Titel Smrt fasizmu spielt auf den einstiger Partisanengruß »Smrt fasizmu svobodo narodu« (Tod dem Faschismus - Freiheit dem Volk) an und der Song »Nemoj biti fasista « (Du sollst kein Faschist werden) befasst sich mit dem Zweiten Weltkrieg. Vordergründig ist dieser Song ein Liebeslied, in dem sich das Mädchen eines Bosniaken einem blonden »Hitlersohn« hingibt. Zwischen den Zeilen spielt es jedoch auf die Tatsache an, dass während des Zweiten Weltkrieges ein Teil der Muslime mit den Deutschen sympathisierte (vgl. dazu Glenny 1993: 215). Der Refrain »Du sollst kein Faschist werden, denn ansonsten, Liebste, bringe ich Dich um « (übers. aus dem Serbokroatischen v. d. Verf.) hingegen hat eine zeitbezogene anti-nationalistische Konnotation.

1992 begann die dreijährige Belagerung von Sarajevo. Die meisten verbliebenen Musiker - der Yugo-Rock blieb eine reine Männersache - wurden für die eine oder andere Kriegspartei mobilisiert: Die ursprüngliche Szene zerbrach. Es bildeten sich aber etwa 30 neue Gruppen (vgl. Basin 1997: 21), die allerdings aus Protest gegen den nationalistischen Wahnsinn den globalen Beat ohne jegliche musikalische Bezüge zum Balkan spielten. Diese Szene sammelte sich rund um den Underground-Sender Radio Zid (Radio

2 Die Redewendung »/hr sollt mit uns rechnen« zieht sich geradezu leitmotivisch durch die gesamte Geschichte der jugoslawischen Rockmusik. Sie ist auf einen Song von Dorde Balasevic aus den 1970er Jahren zurückzuführen, in dem beteuert wird, dass man mit der Jugend auch in Zukunft »rechnen « könne. In den 1980er Jahren ironisierte die slowenische Punk-Gruppe Pankrti diesen Sachverhalt, indem sie den "Schwur der Jugend « in eine gegen das sozialistische Regime gerichtete Drohung umfunktionierte. 1992 wiederum organisierte das Zentrum für Antikriegsaktion unter diesem Motto in Beograd ein groß angelegtes Protestkonzert. 
Mauer), der sich bemühte, die unsichtbaren, jedoch zu diesem Zeitpunkt bereits fest etablierten Barrieren zwischen Individuen unterschiedlicher ethnischer und religiöser Herkunft niederzureißen. Zu den beliebtesten Sendungen von Radio Zid gehörten die Ex-Yu Pop Rock Links mit Erinnerungen an die goldenen Zeiten des Yugo-Rock.

\section{Der Yugo-Rock im Exil}

Im Anschluss an die Kriege in Slowenien (1991), Kroatien (1991/1992), Bosnien und Herzegowina (1992-1995) sowie Serbien (1999) sind einige neue Staaten (Slowenien, Kroatien, Bosnien und Herzegowina, Mazedonien) entstanden. Die Kämpfe kosteten schätzungsweise 350.000 Menschen das Leben und zwangen mehr als 3,5 Millionen Menschen zur Flucht. Serbien und Montenegro kündigten 1992 die Gründung der Föderativen Republik Jugoslawien an, die sich als Nachfolgestaat der ehemaligen Sozialistischen Föderativen Republik Jugoslawien verstand. 2003 ersetzte die Staatsverbindung Serbien und Montenegro die bis dahin bestehende Bundesrepublik Jugoslawien.

Dadurch verschwand nicht nur ein Land, sondern auch sein Name. Der nun heimatlose Yugo-Rock wurde zum Soundtrack eines nicht mehr existierenden Staates und fand eine neue Heimat im Exil. So trafen sich beispielsweise ab 1993 in der Berliner Arcanoa-Bar jugendliche Flüchtlinge aus allen Teilen Ex-Jugoslawiens und begannen hier ihre alten Kassetten und LPs zu spielen. "Musik aus Ex-Jugoslawien hält bis heute die junge Generation vom Balkan zusammen«, schrieben Frank Hoffmann und Rüdiger Rossig in ihrem Beitrag über die Berliner Yugo-Rock-Szene: »Rock gegen den Nationalismus der Teiler und Kriegstreiber (Hoffmann/Rossig 1997: 125).

Was in den Kriegsgebieten unmöglich schien, wurde hier täglich praktiziert: Das harmonische Zusammenleben ohne Rücksicht auf religiöse oder ethnische Unterschiede. Die emotionale Klammer dieser Gemeinschaft bildete der Yugo-Rock, wobei darunter nicht mehr eine spezifische musikalische Gattung in Anlehnung an Bijelo dugme verstanden wurde, sondern die gesamte Musikproduktion des einstigen Jugoslawiens. Die meisten Pjesme bratstva i djetinstva (Lieder der Brüderlichkeit und der Kindheit), wie Petar Janjatovic (1993) in Anspielung an den einstigen politischen Slogan »Bratstvo i jedinstvo jugoslovenskih naroda in narodnosti (Brüderlichkeit und Einheit der jugoslawischen Völker und Volksgruppen) seine Anthologie der Rock-Poesie der Sozialistischen Föderativen Republik Jugoslawien 19671991 betitelte, waren allen bekannt und bildeten eine gemeinsame Quelle der Wehmut, des Trostes und der Erinnerung. 


\section{Yugo-Nostalgie in Slowenien}

Dass in der Arcanoa-Bar in Berlin-Kreuzberg unter anderem die alten sozialistischen Feiertage - etwa der Tag der Jugend ${ }^{3}$ oder der Tag der Republik ${ }^{4}$ - Anfang der 1990er Jahre gute Anlässe für eine Fete boten, hatte mehrere Gründe. Sie waren teilweise als Party-Gag gedacht und teilweise mit der Zielsetzung eingerichtet worden, nationalistisch orientierte Jugendliche von einer Teilnahme auszuschließen. Sie können aber auch als ein Zeichen der Yugo-Nostalgie gedeutet werden, die sich zunehmend nicht nur unter den Flüchtlingen, sondern auch in den aus dem zerfallenen Jugoslawien hervorgegangenen Kleinstaaten ausbreitete.

In Slowenien, das sich verhältnismäßig schnell vom zehntägigen Krieg mit der Jugoslawischen Volksarmee erholte, verzeichnete man die ersten, zunächst auf ein Dasein im subkulturellen Underground beschränkten Symptome der Yugo-Nostalgie bereits Anfang der 1990er Jahre. Ab Mitte der 1990er Jahre kam es jedoch auch im Upperground zu einer Neubewertung der Vergangenheit. 1996 hatten laut der Untersuchung Slovensko javno mnenje 1990-1998 (Slowenische öffentliche Meinung 1990-1998) 34,1\% der Befragten vorwiegend gute Erinnerungen an das ehemalige Jugoslawien. 1998 waren es bereits $36,9 \%$ und $88,2 \%$ der Befragten bezeichneten ihr Leben in Ex-Jugoslawien als gut oder sogar sehr gut (Tos 1999: 565/872).

Weniger eindeutig, und auch weniger positiv, fallen allerdings Einschätzungen in Bezug auf die vom sozialistischen Regime protegierte »Einheit und Brüderlichkeit der Völker und Volksgruppen« aus. Hier scheint sich durch die politischen Gegebenheiten ein tiefer Graben zwischen dem slowenischen »Wir« und den »Anderen« aufgetan zu haben, wobei sich dieses slowenische »Wir« - nicht zuletzt unter Berufung auf die Mitgliedschaft in der Europäischen Union - als »besser«, »klüger« und »kulturell höher entwickelt« betrachtet als die abwertend als Balkanesen bezeichneten anderen Völker des ehemaligen Vielvölkerstaates, denen »Unordnung«, »Unredlichkeit«, »Faulheit«, »Wildheit«, »mangelnde Rationalität« und »Rückständigkeit « bescheinigt wird (Velikonja 2000).

In dieses Spannungsfeld ist auch die immer populärer werdende BalkanKultur anzusiedeln. Alte Yugo-Filme und Fernsehserien haben einen Kult-

3 Der Tag der Jugend wurde am 25. Mai, dem offiziellen Geburtstag des Präsidenten Tito, gefeiert.

4 Der Tag der Republik erinnert an den 29. November 1943, als im bosnischen Jajce die provisorische Regierung des künftigen sozialistischen Staates unter der Führung von Josip Broz Tito gegründet wurde. 
charakter; die zwischenzeitlich verpönten Balkanspezialitäten wie Burek oder Cevapcici stehen schon wieder hoch im Kurs, und wer in der Szene auffallen will, fährt eine (serbische) Zastava (Die Fahne), das einzige in ExJugoslawien produzierte Automodell. Auch Konzerte der alten Yugo-RockGruppen sind gut besucht und Tonträger mit Titeln wie Legenden des YugoRock Verkaufsschlager.

Träger dieser Balkan-Kultur sind allerdings nicht nur die älteren Generationen, die sich dem Eskapismus in die Vergangenheit hingeben. Sie wird auch von den Jüngeren, vor allem Studenten und Angehörigen der einst blühenden Alternativszene von Ljubljana gepflegt, die neben dem Konsum von Produkten der einstigen jugoslawischen Popkultur auch die neuen kulturellen Strömungen in den anderen, nun selbständigen Staaten verfolgen und die sich auch selbst mit dem Balkan kreativ auseinanderzusetzen versuchen (vgl. Velikonja 2000). Einstige Hits werden erneut eingespielt und neue gestaltet, teilweise sogar in der Sprache, die man einst Serbokroatisch nannte und die sich nach diversen ethnolinguistischen Säuberungen ins Serbische, Kroatische und Bosniakische spaltete (vgl. dazu Okuka 1998).

\section{Zaklonisce prepeva}

Unter den slowenischen Gruppen, die sich direkt oder indirekt auf das Erbe des einstigen Yugo-Rocks berufen, muss in erster Linie Zaklonisce prepeva/ Skloniste pjeva (Der Bunker singt) erwähnt werden. Dass sich diese Band dem nostalgischen Yugo-Rock verschrieben hat, geht bereits aus der Ikonographie der CD Novo vrijeme, stare dileme (Neue Zeiten, alte Dilemmas) ${ }^{5}$ hervor, einem Mix politischer (roter Stern, sozialistische Fahne, die Angabe der einstigen republikanischen Hauptstädte) und rockmusikalischer Symbolik (die Bezeichnung Yugo-Rock-Caffe in der für das Hard-Rock-Café typischen Schrift). Musikalisch lehnt sich diese CD weitgehend an die heimischen Rock-Klassiker an. Es gibt aber auch andere Inspirationsquellen, vom Blues bis zur volkstümlichen Musik aus den Gegenden südlich des Flusses Kolpa, wo sich laut alltäglichem Sprachgebrauch die imaginäre Grenze zwischen dem »westlichen Slowenien« und dem »Balkan« befinden soll. Auch Texte

5 Der Titel dieser CD spielt an den einstigen Hit »Novo vrijeme« (Neue Zeiten) der Kultband des slowenischen Undergrounds Buldozer (Bulldozer) aus der Mitte der 1970er Jahre an. Eine besondere Brisanz erhielt dieser Song durch die Zeilen »Neue Zeiten. Alter Zustand. Neue Zeiten. Selbe Scheiße«, da sie als Kommentar zum herrschenden politischen Klima nach Titos Tod im Jahre 1980 verstanden wurden und in dieser Funktion den Eingang in die Umgangssprache fanden. 
dieses Tonträgers sind mehrheitlich in Serbisch bzw. Kroatisch bzw. Bosniakisch verfasst ${ }^{6}$, und sie sprechen eine eindeutige politische Sprache. Beispiele dafür bilden die eigenwillige Coverversion des Songs »Racunajte na nas « (Ihr sollt mit uns rechnen), eine pathetische Hymne an das politische Erbe des verstorbenen Präsidenten Tito von Dorde Balasevic, sowie der Titel "Jugoslavija blues « (Jugoslawien Blues), in dem die Schuld am Zerfall Jugoslawiens dem Westen zugeschoben wird.

\section{Zaklonisce prepeva: »Jugoslavija blues«}

Novo vreme, stare dileme.

Niko nije prvi bez malo krvi [...].

Bilo je Slovenaca, bilo je Hrvata, I Tito je bio nas dok nije bilo rata [...].

Jebacemo mater svima, Od Vasingtona7 do Rima. Zivela Jugoslavijo.

Zivi srecna u svobodi, Ljubav nasa nek te vodi, Jugoslavijo.

Smrt Klintonu in Zak Siraku, Skinucemo glavo svaku. Zivela Jugoslavijo.

Zivi srecna u svobodi, Tito putem nek te vodi, Jugoslavijo.

\section{Zaklonisce prepeva: Jugoslawien Blues}

Neue Zeiten, alte Dilemmas.

Keiner kann ohne ein wenig Blutvergießen der Erste sein [...].

Es gab Slowenen, es gab Kroaten, und auch Tito gehörte uns, bevor der Krieg kam [...].

Wir werden die Mütter von allen ficken, von Washington bis Rom. Es lebe Jugoslawien.

Lebe glücklich in der Freiheit, unsere Liebe soll dich führen, Jugoslawien.

Tod dem Clinton und dem Jacques Chirac, wir hauen allen den Kopf ab. Es lebe Jugoslawien.

Lebe glücklich in der Freiheit, Tito soll dich auf deinem Weg führen, Jugoslawien.

(zit. n. dem Begleitheft zur CD; frei übers. aus dem Serbokroatischen v. d. Verf.).

6 Im einstigen Jugoslawien wurden populäre Songs teilweise in der jeweiligen Landessprache und teilweise in Serbokroatisch verfasst. Im Falle des Yugo-Rock protegierte die Schallplattenindustrie vor allem serbokroatische Produktionen, die sich nicht nur in den größten Republiken, Serbien und Kroatien, sondern im ganzen Land absetzen ließen. Ferner wurde ein derartiger Sprachgebrauch auch politisch gefördert, da man sich vom Abbau von sprachlichen Barrieren zugunsten des Serbokroatischen zunächst einen Beitrag zu der politisch verordneten Einheit und Brüderlichkeit jugoslawischer Völker und Volksgruppen und später zum serbisch dominierten »Unitarismus « erhoffte.

7 Die Gruppe imitiert - allerdings in lateinischer Schrift - die phonetische Rechtschreibung des Serbischen. 


\section{Goran Bregovic - der musikalische Botschafter des Balkans}

Im Westen war der Yugo-Rock zu seiner Blütezeit kaum bekannt. ${ }^{8}$ Dass sich die Situation in den 1990er Jahren maßgeblich geändert hat, ist unter anderem darauf zurückzuführen, dass am Balkan-Konflikt, wie man im Westen die Ereignisse um den Zerfall des einstigen Jugoslawiens zu nennen pflegt, auch die internationale Gemeinschaft beteiligt war und deshalb die Auseinandersetzungen eine ausführliche mediale Aufarbeitung erfuhren. Neben dem Kriegsgeschehen wurde auch über andere Aspekte des Lebens in dieser Region, darunter auch über die Musik, berichtet. Eine besondere Rolle spielte in diesem Zusammenhang MTV, das im bosnischen Bürgerkrieg die Rolle eines musikalischen Kriegsreporters übernahm und in seinen Rockumentaries mit dem Titel Sarajevo Special auch alte Yugo-Rock-Titel brachte (vgl. Hujic 1996).

Den wichtigsten Beitrag zur Popularisierung des Yugo-Rock im Westen leisteten allerdings Musiker im Exil, vor allem Goran Bregovic, der einstige Bandleader der Gruppe Bijelo dugme. Bregovic wurde im Westen zunächst durch die Musik zu den Filmen seines Landsmanns Emir Kusturica' Times of the Gypsies (1989), Arizona Dream (1993) und Underground (1995) bekannt. Später tourte er mit seiner Wedding and Funeral Band, bestehend aus einem Symphonieorchester, einem Männerchor, vier Vokalsolistinnen und einer (Roma-)Blaskapelle, die inm mit den Tales And Songs From Weddings And Funerals auch in diesem Bereich einen internationalen Durchbruch sicherte. Ferner macht er Musik für das Theater und diverse Bühnenspektakel wie etwa Goran Bregovic's Karmen with a Happy End, das - durchsetzt mit Reminiszenzen an Bizets »Carmen « - das Leben der heutigen Roma in den Vorstädten großer Metropolen thematisiert.

8 Mehr Export gab es allerdings in die Ostblock-Länder, vor allem in die einstige DDR und nach Polen, wo viele Yugo-Rock-Gruppen einen Kultstatus genossen.

9 Auch Emir Kusturica stammt aus Sarajevo. Er war zunächst ein Geheimtipp der Underground Filmszene, später Preisträger renommierter Filmfestivals (Venedig, Berlin) und im Jahre 2005 Juror des Cannes Film-Festivals. Musik spielt in allen seinen Filmen eine Rolle. Sie geht in ihrer Funktion weit über die musikalische Untermalung seiner poetischen, teilweise bizarren und teilweise surrealen Szenen hinaus. Nach dem Bruch mit Bregovic arbeitet Kusturica mit der "new primitiv« Gruppe Zabranjeno pusenje (No Smoking Orchestra) zusammen, in der er gelegentlich auch selbst spielt, insbesondere auf Konzerten in Zusammenhang mit der Promotion seiner Filme. Da Zabranjeno pusenje auch enge Beziehungen zu der slowenischen Gruppe Zaklonisce prepeva pflegt, schließt sich hier ein weiterer Kreis des Balkan-Rock. 
Sucht man nach dem Geheimnis des Erfolgs von Goran Bregovic, dürfen neben der hohen Professionalität vor allem zwei Merkmale als ausschlaggebend angesehen werden, die untrennbar mit dem Begriff »Balkan« verbunden sind: Das erste ist der massive Rückgriff auf volksmusikalische Traditionen, nicht nur des ehemaligen Jugoslawiens, sondern der gesamten Balkanhalbinsel. Diese zeichnen sich durch eine reiche Abfolge musikalischer und kultureller Überschichtungen bzw. eine bunte Mischung westlicher und östlicher, byzantinischer und abendländischer, orientalischer und europäischer Elemente aus. Außerdem wurde diese Region trotz Bemühungen sozialistischer Regime nie vollständig industrialisiert (vgl. dazu Hösch 2004: 111), so dass sich dort noch viele traditionelle Lebensgewohnheiten erhalten haben. Hochzeiten und Beerdigungen, die Bregovic zu einem der thematischen Schwerpunkte seiner Arbeit macht, gehören deshalb in diesem Umfeld zu den Höhepunkten des gemeinschaftlichen Lebens und bilden zugleich auch die wichtigsten musikalischen Ereignisse (vgl. Brougthon 2000). Der zweite Balkan-Bezug muss auf einer metaphorischen Ebene gesucht werden. Diese impliziert das Aufeinanderprallen schroffer Gegensätze, die sich sowohl in der Landschaft selbst als auch in den Charaktereigenschaften seiner Bewohner widerspiegeln sollen (vgl. Cuddon 1967: 9; Libal/von Kohl 2000: 50). Goran Bregovic bestätigt:

»Ich finde, wir sind ein bisschen wehleidiger als andere Europäer. Und ich finde, wir sind manchmal zu sehr gefühlsbetont. Wir bewegen uns eigentlich nur in Extremen, entweder es gibt zu viel Freude oder eben zu viel Tränen, aber nichts dazwischen« (Goran Bregovic, zit. n. Buhre 2005).

Dieselben Widersprüchlichkeiten finden sich in der Musik von Goran Bregovic wieder. Hier wechseln mächtiger Blaskapellensound und dichte StreicherArrangements mit harten Drums, weicher Hirtenflöte oder leisen Geigensoli, und die traditionelle bulgarische Vokalpolyphonie mischt sich mit rockigen Gitarrenklängen und bewegten Rhythmen volkstümlicher Percussion-Instrumente. Diese teilweise pathetische Melange mag sich zwar für einige Kritiker scharf an der Grenze zum Kitsch bewegen, sie weckt aber musikalische Assoziationen an den Topos Balkan auch bei jenen, für die dieser keine reale Region, sondern lediglich eine mentale Repräsentation darstellt:

"Alles fließt ineinander, stets getragen von den emotionalen Extremen des Balkans: himmelhoch jauchzend oder zu Tode betrübt. Dazwischen existiert nichts« (www.laut.de/wortlaut/artists/b/bregovic_goran/biographie/index.htm; Zugriff: 8.7.2006).

»Zu viele Krisenmeldungen haben bei der einen oder dem anderen vielleicht vergessen lassen, wofür >Balkan` auch stehen kann: für das Leben in einer 
Authentizität, wie sie suns in Westeuropa r vielfach abhanden zu kommen scheint. Das neuste Werk des ehemaligen Rock-Stars, Schauspielers, Komponisten, Arrangeurs und Produzenten Bregovic ist in genau diesem Sinne >Balkan at its best $\_$! Eine Platte für jeden Moment, weil jeder Moment auf ihr festgehalten scheint. Jede noch so traurige Stimmung findet hier ihren musikalischen Ausdruck; jede noch so überbordende Lebensfreude ihre entsprechende Umsetzung " (www.amazon.de/gp/product/B000068PGX; Zugriff: 8.7.2006).

»Diese Musik verströmt neben der Ruhe aber auch die unbändige Energie des Balkans, so wie die Donau an ihrer Mündung ins schwarze Meer nicht wie ein reißender Gebirgsbach springt, sondern majestätisch, aber mit ungeheurer Macht dahin gleitet« (www.amazon.de/gp/product/B000024969; Zugriff: 8.7.2006).

\section{Weitere Vertreter des Balkan-Rock}

Dass sich Bregovic schonungslos der reichen musikalischen Ressourcen des Balkans, darunter auch des urheberrechtlich nicht geschützten volksmusikalischen Materials, bediente, war oft ein Gegenstand der Kritik. Es bleibt jedoch festzuhalten, dass er durch seine Reinterpretationen die musikalische Überlieferung des Balkans salonfähig gemacht, zahlreiche in- und ausländische Gruppen beeinflusst ${ }^{10}$ und vielen Musikern dieser Region eine Tür zu den westlichen Märkten geöffnet hat. Zu den Profiliertesten unter ihnen zählt das Boban Markovic orkestar aus Serbien - auch dieses Ensemble wurde im Westen zunächst über die Filme Underground und Arizona Dream von Emir Kusturica bekannt (vgl. Maksimovic 2003: 23). Der TrompetenVirtuose Markovic, gefeiert als »König aller Blechbläser «, leitet eine traditionelle Roma-Blaskapelle, in der sich die Überlieferung ottomanischer Militärkapellen mit musikalischen Einflüssen unterschiedlichster Provenienz, von der Volksmusik benachbarter Ethnien, über klassische Märsche bis zu Jazz, Kletzmer und zeitgenössischer Popmusik verbindet. Wie bei anderen Roma-Kapellen dieser Region muss deshalb auch beim Boban Markovic orkestar das Typische nicht im Repertoire, sondern in einer spezifischen Art der Interpretation gesucht werden. »Roma are the best musicians, because they play music of all nations «, erklärte ein Musiker. Und ein anderer ergänzte: »We do not care, whether it [the music] is Turkish, Serbian or Albanian. We just play it livelier« (zit. n. Pettan 2002: 224-225).

10 In Polen löste Bregovic einen echten Folk-Boom aus und wird von den Fans (irrtümlicherweise) als einer der bekanntesten polnischen Musiker angesehen. 
Dies trifft auch auf die Musik von Boban Markovic zu, dessen Kapelle eigenen Angaben nach »Musik aus der ganzen Welt « (zit. n. Maksimovic 2003: 23) spielt: Die Titel der CDs Cigansko srce (Zigeuner Herz), Zlatna truba (Goldene Trompete) oder Balkan Brass Fest verweisen jedoch auf die tiefe Verwurzelung in ihrem Heimatland. Sie zeichnen sich durch energetischen Drive, Virtuosität und die Eigenwilligkeit aus, unterschiedliche musikalische Ideen zu einem leidenschaftlichen improvisatorischen Reigen zu verbinden. Abrupte Brüche im Stil oder unerwartete Übergänge von dem im manischen Tempo gespielten Tutti-Bombast in elegisch-süßliche molltonale Solos sorgen für ein Wechselbad der Gefühle, bei dem - ebenso wie bei den Stücken von Goran Bregovic - »überschäumende Lebensfreude und tiefe Melancholie dicht nebeneinander « liegen (HR Online: Mikado - CD der Woche, 21.-26. November 2005, zit. n. www.piranha.de/records/deutsch/all_g1901.htm; Zugriff: 8.7.2006).

In ihrer Wirkung zwar ähnlich, jedoch musikalisch vollkommen anders gelagert, ist das musikalische Ausdrucksrepertoire von Boris Kovac \& LaDaABa Orchest. Der aus dem serbischen Novi Sad stammende Philosoph, Komponist - er schrieb auch Musik für zahlreiche Theaterstücke -, Saxophonist und Multimedia-Künstler mit starker Affinität zu Literatur und Theater (vgl. Kremer 2006) verarbeitet die folkloristischen Bausteine seiner Musik auf eine individuelle Art und Weise. Das vielschichtige Klangspektrum seiner eleganten Stücke ist urban überformt und gewürzt mit kabarettistischer Ironie. Es ist gleichzeitig zeitgenössisch und archaisch und fusioniert Jazz mit Volksmusik, Avantgarde-Rock, diversen Tanzformen und klassischen Anleihen, teilweise mit Berührungspunkten zum Minimalismus eines Steve Reich oder Phil Glass, sowie Bruchstücken experimenteller Poesie. Auf der Bühne bilden schauspielerisch bzw. tänzerisch konzipierte Performances eine zusätzliche Dimension.

Auch Kovac lebte in den 1990er Jahren zeitweilig im Ausland und befasste sich ausführlich mit den jugoslawischen Bürgerkriegen. Die Abkürzung LaDaABa steht für La Danza Apocalyptica Balcanica: Der apokalyptische Tanz bezieht sich auf die NATO-Bombardements Serbiens 1999. In ihren Projekten The Last Balkan Tango und Ballads At The End Of Time setzte das multiethnisch zusammengesetzte Salon-Orchester die Atmosphäre jener Zeit in einen Zyklus von Standardtänzen (Tango, Walzer, Calypso, Rumba) um, durchsetzt mit Fragmenten musikalischer Volksüberlieferung. In der Kurzbeschreibung der CD Ballads At The End Of Time auf den Internetseiten des Onlinehändlers Piranha Shop heißt es:

»Im zweiten [...] Teil der LaDaABa Serie lässt Boris Kovac noch einmal die ganze Sehnsucht, den ganzen Schwung, die ganze Seele des Balkan aufleben: 
alles, was diese Region so groß gemacht hat, was sie gleichzeitig zerstört hat - von der Eleganz Wiens über den Reichtum Istanbuls bis hin zur Selbstzerfleischung Jugoslawiens in ewiger Unkenntnis über die eigene Identität« (www.piranhashop.de/cgi-bin/shop.cgi?action=showmedium;id=7299; Zugriff: 8.7.2006).

Das Sandy Lopicic orkestar stammt aus Graz. Lopicic verbrachte seine Kindheit in Esslingen, studierte Klavier in Sarajevo und anschließend an der Jazzabteilung der Grazer Kunstuniversität. Hier lernte er auch die meisten Mitglieder seines 1998 gegründeten Ensembles kennen: Viele von ihnen stammen aus den Nachfolgestaaten des ehemaligen Jugoslawiens und kennen sich mit den dortigen volksmusikalischen Traditionen bestens aus. Auch die Musik von Lopicic, maßgebend getragen von den charakteristischen Stimmen der drei Sängerinnen, bildet eine Verschmelzung diverser Stilrichtungen, wird aber in der Regel als Balkan-Jazz bezeichnet. Die CDs Border Confusion und Balkea, beide eine »wilde Mischung von Besinnlichem, vor Freude Überschäumendem, Traurigem und Kraftvollem « (Samar Ertsey in Highlightzone 6/2004, zit. n. www.lopicic.com/press.html; Zugriff: 8.7. 2006), spielen zwar auf die politische Situation auf dem Balkan bzw. in ExJugoslawien an. Doch die oft als »Versöhnungskapelle « gepriesene Formation versteht sich, eigenen Angaben nach, nicht als eine "Politband«, obwohl ihre Mitglieder »auf globaler Ebene natürlich ein klares politisches Verständnis haben«. »Wir massakrieren uns nicht«, erklärt eine der Sängerinnen, »sondern schöpfen aus unserer Kultur« (zit. n. Balkan Fever 2004).

Ferner gibt es einige Balkan-Gruppen, deren Mitglieder nicht mit dem ehemaligen Jugoslawien assoziiert werden können. Zu den bekanntesten unter ihnen gehören die Balkan Playboys aus Budapest. Sie scharen sich um den aus Bulgarien stammenden Multi-Instrumentalisten Nikola Parov, der sich bereits in den 1980er Jahren mit der traditionellen Musik des Balkans befasste und mit der akustisch spielendenen Gruppe Zsaratnok diverse thematische Alben einspielte: Die farbenreichen Arrangements basieren zum Teil auf eigenen musikethnologischen Untersuchungen. Sein zweites Interessengebiet ist der irische Folk - er arbeitete unter anderem mit der Showgruppe Riverdance zusammen -, wodurch es zu einer fruchtbaren Verknüpfung beider Überlieferungen kam. 2003 legten Parov und seine Balkan Playboys mit den CDs Balkan Syndicate und Balkaninis weitere Bekenntnisse zu dieser Region ab.

In New York machte in diesem Zusammenhang vor allem die Gruppe Balkan Beat Box von sich reden. Ihre rauschenden Events, getragen von kostümierten Artisten und begleitet von einer optischen Flut rastlos geschnittener Videos, sind eher künstlerische Performances als Partys und zeichnen 
sich durch einen bunten musikalischen Mix aus, in dem elektronische Dance Music mit volksmusikalischen Elementen des Mittelmeerraumes gekreuzt wird. Inszeniert werden diese turbulenten Spektakel von zwei gebürtigen Israelis, dem Rock-Drummer Tamir Muskat und dem Saxophonisten Ori Kaplan. Kaplan, der sich unter anderem an Avantgarde-Projekten aus dem Umfeld der Knitting Factory beteiligte, spielte auch in Kletzmer Ensembles und in diversen Underground Bands - darunter auch bei Gogol Bordello, die mit ihrem Gypsy Punk Cabaret einen wahren Boom der osteuropäischen Musik in New York auslösten.

Etwas näher an den Balkan-Roots ist die Gruppe Kultur Shock aus Seattle, in der ein gebürtiger Bosnier und ein Bulgare den Ton angeben, und die traditionelle Balkan-Sounds mit Punk, Metal, Funk und Rap zu einer Art alternativer Balkanmusik verbinden.

\section{Drehscheiben musikalischer Kommunikation}

Trotz einiger Ansätze in den USA ist zurzeit der Balkan-Rock (noch) eine weitgehend europäische Angelegenheit. Neben Frankfurt mit DJ Shantel und seinem Bucovina Club scheint Berlin eine wichtige Drehscheibe musikalischer Kommunikation zu sein. Einen Kristallisationspunkt bildet das BalkanBeats Culture Recycling Projekt, die Fortführung der bereits angesprochenen Musikabende für Flüchtlinge aus Ex-Jugoslawien in der Arcanoa-Bar. Auf der ständigen Suche nach immer größeren Locations finden seit 2001 die BalkanBeats Partys im Berliner Mudd Club bzw. in der Kulturbrauerei statt, medial unterstützt von Radio MultiKulti Berlin und einigen Zeitschriften. Unter den zwischen Ironie und Nostalgie anzusiedelnden Mottos wie »Rock den Balkan« oder »Wir gehören Tito, Tito gehört uns« bringen drei aus dem ehemaligen Jugoslawien stammende DJs das Publikum zum Tanzen, und sie laden Live-Acts ein, die zum großen Teil aus Ex-Jugoslawien stammen. Ein weiteres Betätigungsfeld eröffnete sich durch die Herausgabe der CD Balkanbeats mit Stars (Goran Bregovic, Boban Markovic) und Newcomers (Magnifico \& Turbolentza aus Slowenien, Besh O Drom aus Ungarn, Sania \& Balkanika aus Serbien) des Balkan-Rock auf dem neu gegründeten Eastblock Music Label sowie Gastspiele des DJ-Teams in Deutschland, in den Nachfolgestaaten des einstigen Jugoslawiens und neuerdings auch in den USA.

Im Vergleich zu den frühen 1990er Jahren gibt es allerdings gravierende Veränderungen. Das Publikum internationalisierte sich weitgehend, so dass auf den Partys neben Flüchtlingen und Gastarbeitern aus dem ehemaligen Jugoslawien auch viele deutsche Besucher anzutreffen sind. Das Repertoire, 
das sich zunächst auf den Yugo-Rock mit den Schwerpunkten Rock, Punk, Industrial und New Wave beschränkte, wurde insbesondere nach dem Durchbruch von Goran Bregovic und Emir Kusturica durch Ethno-Klänge dieser Region und schließlich durch ein breites Spektrum der Musik aus dem ehemaligen Ostblock erweitert. Schließlich verloren die BalkanBeats-Abende allmählich den Charakter eines »Überlebungstraining[s] von Emigranten « (BalkanBeats 2006) zugunsten von Feten, auf denen Spaß und Tanz im Mittelpunkt stehen.

BalkanBeats Culture Recycling beteiligt sich ferner an der balkanblackbox, einem in Berlin stattfindenden Festival für Film und Kultur aus und über Südosteuropa, insbesondere über das ehemalige Jugoslawien. Gewidmet vorwiegend der unabhängigen Kulturproduktion, wechseln bei diesem Festival kulturelle Veranstaltungen (Musik, Theater, Literatur, Bildende Kunst) mit bisweilen durchaus polemisch geführten Diskussionsforen, in denen aktuelle (kulturpolitische) Tendenzen in den sich wandelnden Gesellschaften auf dem Balkan behandelt werden.

Eine wichtige Einzelpersönlichkeit dieser Szene ist Rüdiger Rossig, einer der Gründer von BalkanBeats und dort auch als DJ tätig. Rossig studierte Balkanologie und Südosteuropäische Geschichte und ist als Redakteur bei der taz tätig. Zwischen 1995 und 1997 arbeitete er als Journalist bei den Vereinten Nationen in Zagreb und Sarajevo und war 1998 Technischer Leiter der OSZE-Wahlbeobachtermission in Sarajevo. Auf seiner Homepage balkanrock findet sich neben einem Sarajevo-Tagebuch über den Einsatz internationaler Organisationen nach dem Dayton-Abkommen in Bosnien eine kurze Darstellung der Geschichte des Yugo-Rock sowie eine Hommage an den verstorbenen Präsidenten Tito (Rossig 2006).

Eine weitere Drehscheibe für die Promotion des Balkan-Rock ist Wien, wo 2004 das erste Balkan Fever-Festival einen Riesenerfolg feierte. »WorldMusic und Balkan-Jazz soll eine ständige und feste Institution in Wien werden«, betonte der künstlerische Leiter und plädierte für regelmäßig abzuhaltende Kulturveranstaltungen über den Balkan. »Es war längst fällig, in der nördlichsten Metropole des Balkans ein Festival aufzuziehen und gegen die herkömmlichen Klischees anzukämpfen« (zit. n. Balkan Fever 2004). Neben Musik mit Größen wie Ivo Papasov, dem ungekrönten »König« der bulgarischen Hochzeitsmusik, der mazedonischen Brassband Original Kocani orkestar, der »Queen of the Gypsies« Esma Redzepova, dem Grazer Sandi Lopicic orkestar sowie dem serbischen Anti-Nationalisten und Kriegsgegner Rambo Amadeus wurde bei diesem Festival auch Literarisches angeboten. Darunter die (musikalische) Lesung von Richard Schuberths Freitag in Sarajevo, einer schwarzen Komödie, die schonungslos mit der »Präpotenz 
und Impotenz jener Kulturschickeria ab[rechnet], die sich als selbsternannte Kreuzritter abendländischer Zivilisation einfindet, wo immer es nach Pulverdampf und Blut riecht« (ebd.). Die Schirmherrschaft über dieses Festival übernahm Wolfgang Petritsch, der einstige österreichische Botschafter in Beograd, EU-Vermittler in Kosovo, oberster internationaler Zivilverwalter in Bosnien und Herzegowina sowie Autor von mehreren Veröffentlichungen über diese Region.

\section{Der Balkan als emotionales Territorium}

Zusammenfassend kann Folgendes festgestellt werden:

1. Der Balkan-Rock ist kein rein musikalisches Phänomen, sondern muss im Zusammenhang mit gravierenden Veränderungen im politischen Umfeld gesehen werden. Wie sich einerseits aus der Häufung von balkanbezogenen (Musik-)Events und andererseits aus den expliziten oder impliziten Bezügen zu den politischen Ereignissen ableiten lässt, bildeten die Bürgerkriege im ehemaligen Jugoslawien einen wichtigen Katalysator für das Aufkommen der Balkan-Welle.

2. An der Konstruktion des Balkan-Fiebers beteiligen sich Akteure mit unterschiedlichem kulturellen, sozialen und politischen Hintergrund (in ExJugoslawien ansässige Ex-Jugoslawen, Ex-Jugoslawen in der Diaspora, Musiker aus den anderen Balkanstaaten, Musiker, Journalisten und Promoter aus den westlichen Industrieländern etc.), die sich - getrieben von unterschiedlichen Motiven - mit dem Thema Balkan auf unterschiedliche Art und Weise auseinandersetzen. Dementsprechend sprechen die Balkan-Events auch unterschiedliche Publika an. In der Bundesrepublik Deutschland gehören dazu sowohl Flüchtlinge und Gastarbeiter aus dem ehemaligen Jugoslawien als auch ein sich als elitär empfindendes deutsches Publikum, das Konzerten in etablierten Locations (Kölner Philharmonie, Alte Oper Frankfurt) beiwohnt oder (vorwiegend junge) Disco-Besucher, die auf den wilden Balkan-Partys abtanzen wollen.

3. Rein musikalisch lassen sich die unter dem Oberbegriff Balkan-Rock subsumierten Phänomene nur bedingt auf einen gemeinsamen Nenner bringen. Eine wichtige Konstante bildet zwar die Berücksichtigung (volks-) musikalischer Traditionen des gesamten Balkanraumes, insbesondere der Roma. Dieser Einfluss verleiht dem Balkan-Sound nicht nur den spezifischen Drive, sondern hat auch eine symbolische Bedeutung. Hierzu die »Gypsy-Queen« Esma Redzepova: 
»Wir sind ein friedliebendes Volk. Wir haben keinen Staat und wir kämpfen nicht darum, ein eigenes Land zu haben. Wir leben in der Welt verstreut. Die Roma sind das einzige Volk, das noch nie einen Krieg gegen ein anderes Volk geführt hat. Wir haben noch nie ein fremdes Land okkupiert, sondern uns immer friedlich darin aufgehalten. Ich finde, dafür hätten wir den Friedensnobelpreis verdient« (Esma Redzepova, zit. n. Balkan Fever 2004).

Auf welche Art und Weise diese Traditionals bearbeitet werden, ist allerdings von Gruppe zu Gruppe und teilweise sogar von Stück zu Stück unterschiedlich. Das Spektrum reicht von landesspezifischen Singweisen und Spieltechniken, teilweise auf Originalinstrumenten, über die Darbietung bekannter Weisen in neuen Arrangements, unterschiedlichsten Fusionen und Crossovers (»Balkan Rumba«, »Balkan Blues«, »Balkan Jazz«, »Balkan Boogie«, »Balkan Hot Step«), über die Gestaltung von hybriden Musikformen, die sich einer herkömmlichen Klassifizierung entziehen, bis zu Eigenkompositionen mit indirekten Bezügen zur Volksmusik im Sinne der folklore imaginaire.

Einen wichtigen Bestandteil der Recyclingmasse bildet ferner das große Reservoir der jugoslawischen Popmusik. Es gibt zahlreiche RevivalBands wie etwa die Balkan Rock Legends, eine Gruppe mit ständig wechselnder Besetzung renommierter Musiker aus Ex-Jugoslawien, die mit ihren Coverversionen in erster Linie den urbanen Teil der sich selbst als New Immigrants bezeichnenden Ex-Jugoslawen im Westen bedient. Ein weiteres Beispiel ist die nach der einflussreichen ex-jugoslawischen Tonträgerfirma benannte polnisch/kroatisch/serbische Gruppe Yugoton, die sich auf das Covern der jugoslawischen Punk-Standards spezialisiert hat. Viele andere Gruppen schreiben allerdings neue Stücke, die mit Zitaten und fragmentarischen Rekontextualisierungen arbeiten oder sich lediglich von ihrem Gestus her an die Vorbilder anlehnen. Dazu kommt noch all das, was die medialen Soundscapes dieser Region hergeben und sich dank der ständigen akustischen Präsenz im Gedächtnis der Musiker und der Fans als musikalisches Wahrzeichen einer Region festgesetzt hat. Die Retro-Ästhetik des Balkan-Rock basiert somit auf freien Anleihen aus diversen Quellen, bei denen das Archaische oft durch experimentelle Arrangements, postmoderne Rekonfigurationen, elektronische Verfremdungen und ironische Brechungen musikalischer Balkan-Klischees einen aktuellen Anstrich bekommt. Die Anwendung heterogener, zwischen Adaption und Dekonstruktion oszillierender und bislang teilweise als widersprüchlich betrachteter musikalischer Bausteine ist Programm, denn, wie Goran Bregovic erklärt, gibt es »in dieser Musik [...] keine Reinheit, 
da ist alles irgendwie bastardisiert « (zit. n. Lehmann 2004: 11). Die musikalische Balkan-Welle funktioniert deshalb als ein weitgehend offenes System, das problemlos unterschiedliche Einflüsse absorbiert, sich in einem ständigen Transmutationsprozess befindet, viel Raum für individuelle Reinterpretationen zulässt und dennoch vor allem in seiner Wirkungsweise einen gewissen Wiedererkennungswert besitzt.

4. Auf dem Balkan selbst, d.h. in diesem Zusammenhang auf dem Gebiet des ehemaligen Jugoslawien, wurden der Begeisterung über die neue/ alte musikalische »Balkan-Ökumene ${ }^{11}$ zunächst Grenzen gesetzt, denn dort suchten vor allem die nationalistischen Führungseliten nicht nach dem Verbindenden, sondern nach dem Trennenden. Events wie z.B. das im Jahre 2005 in Ljubljana durchgeführtes balkanart festival, das sich der Zusammenarbeit der jungen osteuropäischen Kunst- und Medienkultur widmete (Video, Film, Fotographie, Bildende Kunst, Musik mit den Schwerpunkten Ethno, Rock, Jazz und improvisierte Musik, Clubkultur) und sich dabei expressis verbis zur »Betonung der intellektuellen und spirituellen Identität des Balkans« bekannte (vgl. balkanart 2005), bilden deshalb noch eine Ausnahme und haben nach wie vor den Beigeschmack einer politischen Provokation.

So mögen die Fans zwar Erinnerungen an einen gemeinsamen Schatz von Liedern der Brüderlichkeit und der Kindheit (Janjatovic 1993) teilen, die ersten gemeinsamen Konzerte von Gruppen aus den einstigen Republiken Ex-Jugoslawiens fanden jedoch erst 1993 auf neutralem Terrain in Berlin und in Prag statt. In Slowenien spielen serbische Gruppen seit 1993/94 wieder, und 1998 trat der Anti-Nationalist Rambo Amadeus als erster serbischer Musiker wieder in Sarajevo auf. Das Eis schien allerdings erst im Jahr 2005 gebrochen zu sein, als sich die Mitglieder der YugoRock-Legende Bijelo dugme um Goran Bregovic für eine Revival-Tour in Serbien, Kroatien sowie Bosnien und Herzegowina zusammenfanden und auf dem Beograder Hippodrom gemeinsam mit 250.000 Fans ihr Comeback feierten. „Geht es nach den Besuchern der Revival-Konzerte der exjugoslawischen Kultband Bijelo dugme, dann sind Serben, Kroaten und Bosnier wiedervereinigt - zumindest als Fans «, vermerkte Rüdiger Rossig (2005: 16) in der taz. »Der Krieg ist vorbei! Und der weiße Knopf ist das Symbol des Friedens. «

5. Für die westlichen Rezipienten hat allerdings das Balkan-Fieber eine vollkommen andere Bedeutung. In Rezensionen zum Balkan-Rock werden zwar in der Regel auch Bezüge zu den jugoslawischen Bürgerkriegen her-

11 Begriff geprägt von Svanibor Pettan in einem Gespräch mit der Verfasserin. 
gestellt, die meisten Fans aber scheinen mit Balkan-Rock vor allem tanzbare Party-Musik mit einer gewissen eskapistischen Funktion zu assoziieren. "It's a pleasure for all the people coming out of Yugoslavia«, meint ein Student aus Potsdam, der regelmäßig den BalkanBeats-Abenden beiwohnt: »For Germans, it's an adventure«. Und:

»For the diverse Eastern European communities in Germany's capital, the music of their youth binds them together [...]. For the Germans who populate club nights like Balkanbeats [...], the music is an escape into something both familiar and exotic« (zit. n. BalkanBeats 2006).

6. Mit der Betonung von Sachverhalten wie Abenteuer und Exotik knüpft die Rezeption des Balkan-Rock an ältere Wahrnehmungsmuster des Balkans an. Man denke z.B. an die Turquerien, in denen im 17. und 18. Jahrhundert die gehobenen Schichten des Westens ihre Faszination für den Orient ausdrückten (vgl. Libal/von Kohl 2000: 40), an abenteuerliche Reiseberichte, die philhellenische Bewegung, die unter anderen Lord Byron in ihren Bann zog und in Childe Herold's Pilgrimage eine literarische Umsetzung fand (vgl. Todorova 1999), oder an popkulturelle Klassiker wie Karl Mays In den Schluchten des Balkan oder den Kultfilm Mord im Orientexpress. In diesen Werken wird der Balkan als eine »exotische und imaginäre Sphäre konstruiert - ein Ort für Legenden, Märchen und Wunder « (ebd.: 31). Er diente als eine »Projektionsfläche für die revolutionären Sehnsüchte im Westen Europas« (Wagner 2003: 21), bot der romantischen Phantasie »Alternativen « zu der »prosaischen und profanen Welt des Westens « und wurde dadurch zu einer Art »Utopia« bzw. zu einem »Symbol für Freiheit« (Todorova 1999: 31).

Eine derartige romantische Verklärung des Balkans ist gegen das Selbstbild des »europäischen Europäers« (ebd.: 267) gerichtet, der "Sauberkeit, Ordnung, Selbstbeherrschung, Charakterstärke, Gespür für das Gesetz, Gerechtigkeit und effiziente Administration symbolisiert « und sich folgerichtig auf einer »kulturell höher stehende[n] Entwicklungsstufe « (ebd.: 175-176) als die so genannten Balkanesen befinden sollte. Da aber Diskurse stets als Doppelagenten funktionieren, entsprang auch die »Dämonisierung« (ebd.: 31) dieser Region demselben Jahrhunderte alten Ringen um Werthaltungen: Als dionysischer Gegenpol des sich apollinisch gebenden Europas steht der Balkan für das »Verbotene, Erotische, Weibliche «bzw. für die »dunkle Seite des kollektiven Europas « und bildet deshalb einen »Müllplatz für negative Charakteristika« (ebd.: $31 / 85 / 267)$. 


\section{Laut Malcolm Chapman ist jedoch}

»romanticism [...] not primarily a creative process, but a re-evaluative process. [...] Romanticism is a re-evaluation, in the centre, of peripheral issues. The motivation for the re-evaluation is that those in the centre, who carry it out, benefit from it. [...] If moral and political conditions in the centre are appropriate, this transported peripheral feature can be turned, at the centre, into a fashionable and glamorous rarity « (Chapman 1994: 41).

In Bezug auf das hier behandelte Phänomen zeigt sich dieser Mechanismus insbesondere in der gnadenlosen Kommerzialisierung des BalkanSounds, der zurzeit bereits eine feste Marketingkategorie der Weltmusik darstellt.

8. Eine weitere Ebene der Re-Evaluierung betrifft den Balkan-Begriff selbst. Diversen Rekontextualisierungsprozessen unterworfen, wurde dieses musikalische Phänomen seiner ursprünglichen Bezüge entkleidet. Das Lokale, Regionale wurde transregional und transnational, und es kam zu einer phantasmagorischen Trennung des vorwiegend geographisch determinierten »space « zugunsten des sozial konstruierten »place« (vgl. hierzu Stokes 1994: 3). In diesem neuen Koordinatensystem mutierte auch der zumeist als "Schimpfwort« (Todorova 1999: 264) verwendete Begriff »Balkan« zu einer Metapher, die sich nicht mehr auf eine von Krisen, Krieg und ethnischen Säuberungen gekennzeichnete Region bezieht, sondern ein durchaus positiv konnotiertes »emotionales Territorium« bezeichnet, das sich laut Goran Bregovic (zit. n. Lehmann 2004: 11) irgendwo zwischen Istanbul und Budapest erstreckt.

\section{Literatur}

Anon. (1991/92). »Special Focus: Sarajevo. In: Balkanmedia, Winter, S. 36-43.

balkanart (2005). »balkanart festival.«In: www.terminal00.org/slo.html (Zugriff: 27.3.2005).

Balkan Fever (2004). Balkan Fever - Wien 2004. 1. Internationales Balkan Festival. Pressematerial. Wien: MICA.

BalkanBeats (2006). »BalkanBeats Culture Recycling.« In: www.balkanbeats.de/ introd.htm (Zugriff: 1.7.2006).

balkanblackbox (2005). »Balkanblackbox.« In: www.balkanblackbox.de (Zugriff: 23.12.2005).

Barbabegovic, Peter (1984). „Walterji. Glasbeno pismo« [Walter. Ein Musikbrief]. In: Mladina, 13. Dezember, S. 38-40.

Basin, Igor (1997). "Sarajevo ima kulturo« [Sarajevo hat Kultur]. In: Muska, Nr. 8, S. 20-25. 
Brougthon, Simon (2000). Weltmusik. Rough Guide. Stuttgart u.a.: Metzler.

Buhre, Jakob (2005). „Für die Filmindustrie bin ich zu schlecht. Der bosnische Komponist Goran Bregovic über Filmmusik, Heimatgefühle und die Seele des Balkan. « In: www.planet-interview.de/interviews/pi.php?interview=bregovic-goran (Zugriff: 1.7.2006).

Chapmann, Malcolm (1994). »Thoughts on Celtic Music«. In: Ethnicity, Identity and Music. The Musical Construction of Place. Hg. v. Martin Stokes. Oxford: Berg, S. 28-44.

Cuddon, John Anthony (1967). Jugoslawien. Ein Führer. München: Prestel-Verlag.

Glenny, Misha (1993). Jugoslawien. Der Krieg, der nach Europa kam. München: Knaur.

Gracanin, Tomi (1985). "Ljubljana peva« [Ljubljana singt]. In: Mladina, 12. September, S. 46-47.

Gracanin, Tomi (1987). »Plavi orkestar. Smrt fasizmu« [Das Blaue Orchester. Tod dem Faschismus]. In: Mladina, 13. Februar, S. 44.

Hösch, Edgar (2004). Geschichte des Balkans. München: Verlag C.H. Beck.

Hoffmann, Frank / Rossig, Rüdiger (1997). »)Zählt nicht auf uns`. Jugoslawische Jugendliche in Berlin. «In: Jugendkulturen zwischen Kommerz \& Politik. Hg. v. Klaus Farin. Erfurt: LZT, S. 124-139.

Hujic, Lida (1996). »l hope you're enjoing your party. MTV in wartorn Bosnia.« In: Screen 37, Nr. 3, S. 268-277.

Janjatovic, Petar (1993). Pesme bratstva i detinjstva. Antologija rok poezije SFR Jugoslavije 1967-1991 [Lieder der Brüderlichkeit und der Kindheit. Eine Anthologie der Rock-Poesie der Sozialistischen Föderativen Republik Jugoslawien 19671991]. Beograd: Geopoetika.

Janjatovic, Petar (1998). Ilustrovana YU Rock Enciklopedija [Illustrierte Enzyklopädie der jugoslawischen Rockmusik]. Beograd: Geopoetika.

Kremer, Draga (2006). »Intervju: Boris Kovac, multimedijalni umetnik. Pijani od Panonije« [Interview: Boris Kovac, multimedialer Künstler. Besoffen von Panonien]. In: www.vreme.com/cms/view.php?id=334909 (Zugriff: 1.7.2006).

Lehmann, Oliver (2004). »Goran Bregovic: Natürlich bin ich Träumer.«In: City, 20. Oktober, S. 8-11.

Libal, Wolfgang / von Kohl, Christine (2000). Der Balkan. Stabilität oder Chaos in Europa. Hamburg, Wien: Europa Verlag.

Maksimovic, Dijana (2003). »Boban Markovic. Balkanska truba osvaja svet« [Boban Markovic. Eine Balkantrompete erobert die Welt]. In: Balkanmedia, Estradni magazin, 24. März, S. 23.

Okuka, Milos (1998). Eine Sprache, viele Erben. Sprachpolitik als Nationalisierungsinstrument in Ex-Jugoslawien. Klagenfurt: Wieser.

Pettan, Svanibor (2002). Roma Musicians in Kosovo. Integration and Creativity. Budapest: Institute of Musicology of the Hungarian Academy for Science.

Ramet, Sabrina (Hg.) (1994). Rocking the State. Rock Music and Politics in Eastern Europe and Russia. San Francisco: Westview.

Rossig, Rüdiger (2005). »Weißer Knopf statt roten Sterns. «In: taz, 30. Juni, S. 16.

Rossig, Rüdiger (2006). »Balkanrock.« In: www.balkanrockruediger-rossig.de (Zugriff: 15.1.2006).

Stokes, Martin (1994). »Introduction«. In: Ethnicity, Identity and Music. The Musical Construction of Place. Hg. v. Martin Stokes. Oxford: Berg, S. 1-27.

Todorova, Maria (1999). Die Erfindung des Balkans. Europas bequemes Vorurteil. Darmstadt: Primus Verlag. 
Tomc, Gregor (1998). »Trdozivi jugorock« [Hartnäckiger Yugo-Rock]. In: Delo, 30. April, S. 41.

Tos, Nikola (Hg.) (1999). Vrednote $v$ prehodu II. Slovensko javno mnenje 1990-1998 [Wertewandel II. Slowenische öffentliche Meinung 1990-1998]. Ljubljana: Dokumenti SJM, FDV.

Velikonja, Mitja (2000). »Bivsi domaci. Balkanska kultura na Slovenskem po letu 1991 « [Die ehemaligen Heimischen. Balkankultur in Slowenien nach 1991]. Unveröffentl. Ms.

Virant, Jana (1998). "Longplay. Zavrtite si longplay intervju s Saso Losicem-Loso« [Longplay. Spielen sie das Longplay-Interview mit Saso Losic-Losa]. In: P.S., Juni, S. 25-29.

Wagner, Richard (2003). Der leere Himmel. Reise in das Innere des Balkan. Berlin: Aufbau-Verlag.

\title{
Diskographie
}

Bijelo dugme (1986). Pljuni i zapjevaj, moja Jugoslavijo [Spuck und sing, mein Jugoslawien]. (LP) Diskoton, 8244.

Zaklonisce prepeva (ca. 1997). Novo vrijeme, stare dileme [Neue Zeiten, alte Dilemmas]. (CD) Shelter Records, SRCD 001.

Plavi orkestar (1986). Smrt fasizmu [Tod dem Faschismus]. (LP) Jugoton, LSV 63262.

\begin{abstract}
The musical texture of Balkan Rock is an open system, able to incorporate inspirations from different sources: authentic folk music of the Balkans, especially music of the Gypsies, Rock and Pop music from former Yugoslavia, Jazz, Kletzmer and other musical styles. To a high extend its international dissemination is due to the civil wars in former Yugoslavia, forcing millions of people to exile. Thus the reception of Balkan Rock depends on the background the recipients come from. If for the aficionados from former Yugoslavia Balkan Rock is associated with memories of what used to be a communal country, for the Western fans the same music has rather an escapist function, reconstructing the image of the Balkans as an emotional alternative to the rational concept of Europe.
\end{abstract}

